\title{
Familles, crèches, précarité
}

\author{
Enzo Catarsi
}

La perspective écologique du développement, aujourd'hui de plus en plus partagée, met en évidence la relation existante entre les responsabilités des différents acteurs sociaux, décideurs politiques et spécialistes, par rapport au développement des enfants. Le rapport qui existe entre la croissance des nouvelles générations et le milieu social d'origine semble ainsi évident aussi bien en termes de bien-être global que de succès scolaire et existentiel. Il est donc urgent d'expliciter les conditionnements sociaux du développement pour projeter des interventions capables de combattre dès le départ les inégalités suscitées chez les enfants par leur milieu d'origine et par les relations dans lesquelles ils grandissent. En effet, comme il a été écrit à juste titre, "l'homme est pris, dès sa naissance, dans une série de liens, familiaux d'abord mais déjà, d'emblée, sociaux. Toute vie s'inscrit dans des entrelacs de relations. C'est sur ce fond relationnel que s'effectue le développement de l'enfant... et de sa famille car il ne faut pas perdre de vue que si les parents font l'enfant, celui-ci fait aussi les parents au travers de la relation qui l'unit à eux" (Pourtois, Desmet, Lahaye, 2008, 87).

\section{Conditionnement social et succès scolaire}

En effet différentes enquêtes ont désormais souligné l'importance des conditionnements du milieu. Parmi ces enquêtes les recherches menées depuis trente ans par Jean Pierre Pourtois et son équipe ont montré très clairement l'influence que la famille exerce sur le développement des enfants et l'adaptation de ces derniers à l'école. (Pourtois, 1979). Ces enquêtes ont en même temps mis en évidence comment, à cinq ans déjà, certaines variables familiales déterminent le futur cursus scolaire des enfants (Desmet, Pourtois, 1993). Elles ont souligné clairement le conditionnement de l'origine sociale sur le succès scolaire (Nimal, Lahaye, Pourtois, 2000). Par ailleurs une recherche plus récente a indiqué 
comment, d'une génération à l'autre, il y a "stabilté dans le changement" et, dans le fond, continuité surtout en ce qui concerne la dimension du développement intellectuel et l'insertion sociale (Lahaye, Pourtois, Desmet, 2007).

Différentes enquêtes ont été menées également en Italie par d'illustres sociologues de l'éducation qui ont mis en lumière l'importance de l'origine familiale sur les résultats scolaires, en dénonçant véhément le caractère traditionnel du système scolaire italien. Giorgio Brunello et Daniele Checchi (2005) ont étudié le cursus scolaire d'un échantillon d'Italiens nés entre 1941 et 1970, en précisant que leur réussite scolaire est le fruit de différentes variables, parmi lesquelles le nombre relativement bas d'élèves par rapport au nombre d'enseignants mais plus encore le niveau de formation des parents. Plus généralement les données semblent démontrer qu'une école de qualité plus élevée, surtout grâce au nombre relativement bas d'élèves par rapport au nombre d'enseignants, remplace le niveau d'instruction des parents dans le processus de construction du capital humain individuel. Dans ce cas particulier les auteurs en concluent qu'une augmentation des ressources financières publiques investies dans l'éducation constitue un moyen essentiel pour réduire les différences dues au niveau culturel des parents.

Par ailleurs, les investissements ont de plus en plus diminué en Italie, ces dernières années, et ont fini ainsi par amplifier les résultats sélectionnants du système scolaire italien - encore conditionné par la maladie "gentilienne". Cela est particulièrement visible au moment du choix du type de lycée qui, aujourd'hui encore, est influencé par le milieu social d'origine, comme le soulignent d'un commun accord les sociologues de l'éducation. "Le diplôme obtenu par un jeune - écrit Giancarlo Gasperoni $(2002,75)$ - continue de dépendre de façon non négligeable de ses origines sociales". En effet le jeune tire de sa famille d'origine des ressources matérielles et culturelles. Les ressources matérielles lui permettent en général de pouvoir étudier plus longtemps, jusqu'à l'université, sachant que $50 \%$ des étudiants issus des classes aisées terminent leurs études contre $26 \%$ des enfants de travailleurs indépendants et à peine $17 \%$ des enfants d'ouvriers. Quant aux ressources culturelles elles contribuent à ce résultat comme le montrent les données d'une recherche Iard spécifique. Les jeunes issus de familles culturellement élevées s'inscrivent en effet à l'université dans $60 \%$ des cas contre $12 \%$ des jeunes dont les parents ont un faible niveau d'études.

L'influence du milieu culturel d'origine est déjà visible, par ailleurs, à la fin du collège. En effet, au niveau national, en Italie, presque deux 
tiers des jeunes issus de familles culturellement élevées, réussit le brevet des collèges avec mention, contre $23 \%$ des jeunes du même âge issus de familles moins cultivées. Le milieu social conditionne donc le choix du lycée. Les jeunes issus de familles au niveau culturel moins élevé choisissent, en général, les lycées techniques et - encore plus - les lycées professionnels, alors que les autres préfèrent les lycées considérés, en général, unanimement comme meilleurs (Gasperoni, 2002, 74-80).

Cet avis est partagé par une autre chercheuse qui confirme que l'origine sociale continue d' influencer lourdement le choix des études et - par la suite - la profession même. "Toutes les recherches - écrit Carla Facchini $(2001,21)$ - mettent en évidence que les jeunes issus de familles dont les parents ont un niveau d'études supérieur et de bons revenus, ont tendance à continuer leurs études après l'école contrairement aux jeunes issus de familles dont les parents ont été peu scolarisés et dont les revenus sont peu élevés. En même temps, alors que les premiers préfèrent les lycées, les autres choisissent les brevets techniques et les écoles professionnelles".

Les mêmes recherches réalisées par Diego Gambetta (1990) et Giancarlo Gasperoni (1996) montrent comment les premiers, issus des classes ouvrières, choisissent souvent des filières professionnelles qui permettent d'acquérir plus rapidement une qualification et l'entrée dans le monde du travail. Les enfants de parents plus instruits - et en général avec de bons revenus - s'inscrivent par contre au lycée pour continuer des études universitaires. Pas de changements significatifs par rapport aux années 60/70, même si des jeunes sont placés "différemment" dans le système scolaire par rapport à leur origine sociale. Mais ce sont des cas assez rares qui n'altèrent pas une tendance toujours plus consolidée avec le temps. Cette tendance pénalise de plus en plus les familles les plus pauvres aux petits revenus.

\section{Services pour l'enfance et prévention}

Dans ce contexte, les recherches sur la petite enfance montrent à quel point les premières années de l'enfant sont importantes pour la construction et la formation de la conduite future du sujet. En même temps, le fait que l'enfant soit l'acteur de son développement dans plusieurs contextes de vies, là où il développe des relations interpersonnelles très significatives, est une conviction de plus en plus répandue. En effet cette théorie se fonde sur l'hypothèse que le sujet se forme et restructure sa 
conduite sur la base des expériences vécues dans plusieurs contextes de vie et dans son milieu familial et social environnant. De la même manière, il faut prendre en compte le rôle que l'éducation et les pratiques sociales qui la caractérisent ont sur le développement de l'enfant. Dans cette perspective Chantal Zaouche aborde à juste titre la question de la socialisation et de l'acculturation de la petite enfance en termes de prévention (Zaouche-Gaudron, 2006). En particulier, elle met l'accent sur le besoin d'analyser les différences individuelles des enfants et de travailler sur la formation des parents pour éviter que les modèles éducatifs ne se répètent de générations en générations. Des modèles d'éducation d'autant plus négatifs dans des situations où certaines familles sont définies "vulnérables" ou "à risque" et assimilées, parfois, à des familles considérées comme "dangereuses".

Il existe, par ailleurs, des théories qui analysent l'influence du milieu d'origine de l'enfant surtout des enfants issus de familles culturellement défavorisées (Zaouche-Gaudron, 2005). D’autres recherches réalisées au Canada, prenant en compte des enfants de 4 à 6 ans, montrent comment les caractéristiques de la famille d'origine conditionnent le développement linguistique et en général cognitif de l'enfant et moins le développement psychomoteur (Palacio-Quintin, Terrisse, 1997), comme le soulignent par ailleurs d'autres enquêtes que nous analyserons ultérieurement. Mais les facteurs qui semblent plus déterminants sont les facteurs socio-économiques, comme le montre une étude, très intéressante, réalisée sur les données des six premières années du British Household Panel Study. Il apparaît que le succès scolaire des parents conditionne celui des enfants et qu' une mauvaise réussite scolaire de ces derniers est influencée en particulier par des phénomènes tels que la provenance de familles monoparentales ou avec de faibles revenus et en particulier de familles de chômeurs (Ermisch, Francesconi, 2001; Ermisch, Francesconi, Pevalin, 2004).

D'autre part, les familles avec de plus grandes difficultés ont en général moins de ressources non seulement du point de vue économique mais aussi culturel. Par exemple il a été souligné que d'une instabilité dans les conditions de travail découle une insécurité pour cerner la façon de prendre soin des enfants. Les possibilités, offertes par l'administration publique aux familles avec de faibles revenus, de payer la quote-part familiale réduite, ne permet pas, en réalité, d'avoir des résultat significatifs en ce qui concerne l'accueil des petits enfants. Il faut donc se contenter d'une solution quelconque au détriment de la qualité. Et ceci apparaît encore plus discriminatoire à la lumière des résultats de certaines re- 
cherches qui montrent que fréquenter un service pour l'enfance a des effets positifs sur le développement des enfants (Varin, 2007), en particulier sur ceux qui sont issus des communautés les plus pauvres (Loeb, Fuller, Lynn, Kagan, Carrol, 2004; Votruba-Drzal, Levine Coley, Lindsay Chase-Lansdale, 2004). Donc, c'est également pour ces raisons, qu'il est nécessaire d'étudier plus à fond, l'échec scolaire. En même temps, il convient de mettre en place des parcours de prévention précoce qui passent obligatoirement par la diffusion des services pour l'enfance et la promotion d'une école qui favoriserait les acquis personnels et qui activerait des connaissances.

\section{Education familiale et réflexivité des parents}

Mais ce qui apparait encore plus important est d'activer des programmes de soutien à la parentalité et en particulier des programmes d'éducation familiale, fondés sur l'idée du grand potentiel que possède l'être humain et de la confiance à avoir en ses propres capacités et en ses possibilités de développement. Tout ceci ne doit pas nous faire oublier que l'éducation implicite joue un rôle important et, donc, que les schémas assimilés pendant l'enfance influencent beaucoup le développement, jusqu'au maintien de logiques conservatrices. Il apparait par ailleurs évident qu'il est nécessaire de partir des schémas métabolisés par le sujet pour sauvegarder sa cohérence identitaire.

De la même manière l'éducation familiale a comme objectif de promouvoir l'autonomie du sujet, à même de gérer sa vie et les choix qui le caractériseront. Nous nous rapportons clairement à la notion de empowerment et à la possibilité qui doit être donnée aux personnes d'améliorer leurs conditions de vie (Pourtois, Desmet, Lahaye, 2004). Ces auteurs écrivent à juste titre: "il s'agit de mener des actions suscitant une activité de réflexivité sociale et individuelle, facilitant la résistance aux schèmes opératoires et implicites qui ne sont plus fonctionnels, bref encourageant une prise en charge plus consciente et plus autonome de la situation. On demande ici aux personnes de se produire comme sujets de leurs actes. Cette perspective s'appuie exactement sur les constatations précitées qui ont certes souligné la puissance de l'éducation implicite mais aussi sa capacité à se réélaborer constamment" (Pourtois, Desmet, Lahaye, 2008, 95-96).

En effet, l'intervention socio-éducative a comme objectif de promouvoir l'autonomie et l'autodétermination du sujet, tout en conservant les 
racines historiques et sociales afin d'éviter de dangereuses crises identitaires. Et c'est justement pour cette raison que, en entrant dans la perspective d'une éducation familiale, partir des connaissances antérieures des individus constitue un choix de fond important, afin de mettre en valeur les personnes et mettre les parents dans les conditions d'améliorer les relations avec leurs enfants et de répondre aux besoins de ces derniers. Ceci est surtout valable pour les familles défavorisées qui risquent plus que d'autres de faire peser sur leurs enfants les conséquences de l' "éducation implicite". Dans le cadre des recherches menées par JeanPierre Pourtois et Huguette Desmet à ce sujet, les interviews réalisées auprès de parents issus de milieux défavorisés mettent généralement en évidence que ceux-ci sont intéressés à l'avenir scolaire de leurs enfants. Cependant elles mettent également en évidence que la majorité des parents n'a pas les capacités culturelles pour interagir avec le système scolaire. Il en résulte que ces parents finissent par prendre une attitude d'indifférence et d'éloignement ostentatoire vis à vis de l'institution. Dans ce cas spécifique l'analyse des données relatives aux familles examinées, montre que les familles, où les enfants réussissent à l'école, sont caractérisées par des attitudes "d'encouragement" de la part des parents, qui se concrétisent en "«estime de l'enfant, élaboration d'un projet professionnel à son égard, investissement de l'école, perception positive et professionalisante de celle-ci, désir de voir l'enfant réussir socialement, attachement moins fusionnel, acceptation teintée de restriction, stimulation à l'indépendance» (Pourtois, Desmet, 2004, 210).

Il est donc nécessaire de travailler à la diffusion des programmes d'éducation familiale, même sur la base des résultats intéressants qui ont été obtenus jusqu'à présent. En effet il existe plusieurs projets qui ont été programmés pour améliorer les compétences des parents et pour renforcer une certaine estime d'eux-mêmes (Catarsi, 2001 e 2003a; Barras, Pourtois, 2005). En effet il faut abandonner l'image d'une famille "inadéquate" ou "dysfonctionnante", dans la mesure où chaque famille possède des ressources potentielles qui peuvent être développées. Cette attitude a l'avantage de promouvoir des compétences. C'est pour cette raison que le spécialiste qui travaille avec les parents doit être conscient que son rôle prioritaire n'est pas celui de transmettre un savoir mais bien celui d'aider les personnes à donner le meilleur d'elles-mêmes et de "devenir ce qu'elles sont" (Catarsi 2003b et 2008).

Plus précisément il s'agit de prévoir des occasions de rencontres hebdomadaires en petits groupes (10-15 parents, mais nous savons pertinemment que ce sont en général des mères) où il serait possible d'em- 
mener les petits enfants. Ces derniers seraient suivis par des éducatrices pendant que les mamans discutent entre elles ou avec un "facilitateur" du groupe, qui pourrait être une éducatrice de crèches avec une longue expérience et une préparation adéquate.

En effet le groupe, organisé dans une perspective rogersienne, agit dans une optique de prévention et favorise le bien-être des personnes, et contribue à ce que surtout les parents définis "fragiles" ou carrément "inadéquats" retrouvent ce que l'on appelle "l'estime de soi". «Les rencontres parentales permettent, par la force du groupe - écrivent à juste titre Jean-Pierre Pourtois et Christine Barras (2004, 68) - d'augmenter l'estime de soi et le sentiment que, malgré ses faiblesses, il est possible d'être un parent acceptable. Le discours transmis par les média est un idéal inaccessible, qui enfonce la personne précarisée dans le sentiment qu'elle n'est rien. Renforcer le «moi» du parent permet d'instaurer une dynamique positive qui rejaillit sur tous les membres de la famille».

Il est clair que les programmes de formation des parents qui ont de petits enfants ont un coût. Mais il est tout aussi évident qu'en comparant les coûts et les bénéfices, les bénéfices l'emportent dans la mesure de 3 à 1, comme l'écrit Kevin J. Swick (2009, 405). Il faut aussi rappeler, comme le relève une autre recherche (Lynch, 2004), que les enfants des familles qui participent à ces programmes ont un plus grand taux de réussite à l'école et tombent plus difficilement dans les filets du système judiciaire ayant ainsi plus de chances de devenir de bons citoyens.

\section{Education familiale et participation des parents}

La crèche et les autres services pour l'enfance $-\mathrm{y}$ compris l'école maternelle - peuvent donc devenir des contextes de formation également pour les parents, qui peuvent, entre autre, contribuer à la réalisation d'une formation contextualisée. En même temps il est utile de rappeler que Urie Bronfenbrenner soulignait, il y a plus de vingt ans déjà, que le rapport entre l'école et la famille était important pour une bonne réussite scolaire des enfants. Ce chercheur affirmait que ces procédés sont plus importants pour le développement de l'enfant que les conditions socio-économiques mêmes (Bronfenbrenner, 1986, 300). D'autre part, d'autres recherches ont confirmé cette idée et montrent que l'investissement des parents dans la vie scolaire favorise la réussite scolaire de leurs enfants (Ravn, 1996).

Plus récemment encore, ont été publiés les résultats obtenus avec le programme des Centres des Enfants et des Parents de Chicago. Ce programme 
qui prévoyait expressément la participation des familles, met en évidence que cette participation favorise l'acquisition des compétences scolaires, en commençant par la "lecture", et, en général, la future réussite scolaire des enfants. A ce propos, il est intéressant de signaler que les programmes de formation pour les parents, qui prévoyaient également des visites à domicile, se sont révélés efficaces en diminuant leur isolement (Clement, Reynolds, Hickey, 2004). De la même manière les données analysées montrent que fréquenter les services pour l'enfance est un facteur qui réduit la future délinquance des jeunes, comme l'a déjà précisé, par ailleurs, une recherche précédente (Reynolds, Temple, Robertson, Mann, 2001).

En effet les enfants sont rassurés en voyant que les adultes qui s'occupent d'eux ont de bonnes relations. Ils s'adaptent ainsi à mieux vivre à la crèche et développent des relations positives avec les éducatrices. C'est donc pour cette raison que le travail avec les parents ne peut pas être considéré, à la crèche et à la maternelle (mais en général dans toutes les institutions de formation) comme un élément supplémentaire du curriculum, mais bien comme un aspect fondamental et spécifique.

En effet, la participation des parents dans le fonctionnement des services pour l'enfance italienne est un élément spécifique du projet de ces importantes institutions éducatives. Aujourd'hui il s'agit d'aller bien au-delà: faire en sorte que la crèche et la maternelle deviennent des contextes d'éducation familiale et de formation des parents en réalisant des programmes qui rendent les parents aptes à aider à la maison au développement général de leurs enfants. Il est en particulier souhaitable que les parents soient concernés par des programmes pour le développement linguistique des enfants. A cet égard, il semble que la lecture à haute voix d'albums illustrés, empruntés éventuellement à la crèche ou à l'école, soit particulièrement utile. En effet, plusieurs recherches ont mis en évidence que la lecture faite par les mamans facilite l'acquisition du vocabulaire et le développement du langage, même avec des enfants issus de familles défavorisées et aux faibles revenus (Rebello Britto, Brooks-Gunn, Griffin Source, 2006).

Plus récemment encore, certains auteurs américains ont à juste titre souligné que "les différences individuelles entre les enfants, en ce qui concerne le langage et les premières compétences en début de cursus scolaire, sont dues à la quantité et à la qualité des interactions linguistiques avec leurs parents et au contact avec la langue écrite dans le milieu familial (Russell Carter, Chard, Lull Pool, 2009, 520).

En effet, quand les enfants sont impliqués dans des activités linguistiques propres à la vie quotidienne, ils ont plus de possibilités de dé- 
velopper des compétences et des concepts. C'est justement pour cette raison qu'il semble utile de cerner les moments de la journée où il est plus facile de motiver les enfants dans des activités de lecture, de conversation et également d'écriture, avec les plus grands. Il apparait alors évident qu'il y a moins de possibilités le matin, si ce n'est à l'heure du petit déjeuner ou pendant le trajet pour aller à la crèche ou à la maternelle. Il y aura plus d'occasions dans l'après-midi, une fois l'enfant rentré de l'école, quand il jouera seul ou quand il regardera la télévision avec sa maman. De la même manière il pourra écouter un des parents qui lui lit à haute voix un album illustré et, dans tous les cas, parler pendant le dîner. Le moment du bain de l'enfant sera un moment privilégié pour le stimuler à parler de sa journée, de ses expériences, et interagir ainsi linguistiquement avec un vocabulaire riche et articulé.

Bien sûr à la base du développement il n'y a pas que les interactions linguistiques et c'est pour cette raison que les interventions d'éducation familiale sont importantes. En effet, plusieurs recherches ont montré comment le milieu familial et surtout la qualité des relations entre parents et enfants constituent des éléments annonciateurs significatifs du futur développement de l'enfant. En particulier, ces recherches ont montré que les mamans qui s'engagent avec plus de sensibilité et plus intensément dans la relation avec leurs enfants motivent plus ces derniers et ont des enfants qui développent de plus grandes compétences cognitives, linguistiques et sociales (Tamis-LeMonda, Shannon, Cabrera, Lamb, 2004; Pan, Rowe, Singer, Snow, 2005; Pancsofar, Vernon, Feagans, 2006). De la même manière, une autre recherche réalisée avec des parents d'enfants de 12 mois, montre que le niveau d'amour conjugal annonce le vocabulaire utilisé aussi bien par la mère que par le père, un an après, avec leur enfant de 24 mois. En effet, des relations amoureuses chaleureuses et intenses dans le couple, font que la mère et le père développent une entente très utile aux interactions linguistiques et émotives avec leurs enfants. Donc, des programmes d'éducation familiale bien organisés peuvent faciliter les relations non seulement avec les enfants, mais également à l'intérieur du couple, là où les difficultés et les incompréhensions - consciemment élaboréespeuvent être dépassées et devenir une source pour de nouvelles relations plus fécondes, avec des conséquences positives surtout pour les enfants. En définitive, la crèche et la maternelle peuvent être des occasions significatives pour la croissance globale des enfants et de leurs parents et donc se caractériser comme des instruments fondamentaux pour la promotion d'une société démocratique. 


\section{Bibliographie}

Barras C., Pourtois J.P. (2005): Développer les compétences éducatives des familles en situation de précarité. Empan, n. 4, 2005, pp. 65-73.

Boyer D. (2005): Modes d'accueil de la petite enfance et précarité. Empan, n. 4, 2005, pp. 91-100.

Bronfenbrenner U. (1986): Recent advances in research on the ecology of human development, in Silbereisen R.K. et al. (a cura di), Develompment as action in context. Problem behaviorand normal youth development. Berlin: Springer-Verlag, 1986, pp. 287-309.

Brunello G., Checchi D. (2005): School quality and family background in Italy. Economic of Education Review, n. 24, pp. 563-577.

Catarsi E. (2001) : Experiences d'education familiale en Italie, in Gervilla Castilla A. et al. (dir.), Familia y educacion, vol. II. Malaga: Università di Malaga, pp. 1051-1056.

Catarsi E. (2003a): Essere genitori oggi. Tirrenia (PI): Edizioni del Cerro.

Catarsi E. (2003b): Un "professionista senza camice": l'animatore di educazione familiare, in Catarsi E. (a cura di), L'animatore di educazione familiare: una nuova figura professionale?, Regione Toscana-Istituto degli Innocenti. Pisa: ETS, pp. 15-30.

Catarsi E. (2008) : Pedagogia della famiglia. Roma: Carocci.

Clements M.A., Reynolds A.J., Hickey E. (2004): Site-level predictors of children's school and sociale competence in the Chicago Child-Parent Centers. Early Childhood Research Quarterly, n. 19, pp. 273-296.

Desmet H., Pourtois J.P. (1993): Prédire, comprendre la trajectoire scolaire. Paris: Presses Universitaires de France.

Ermisch J., Francesconi M. (2001): Family matters: impacts of family background on educational attainments. Economica, n. 68, pp. 137-156.

Ermisch J., Francesconi M., Pevalin D.J. (2004): Parental partnership and joblessness in childhood and their influence on young people's outcomes. Journal of the Royal Statistical Society, vol. 167, 1, pp. 69-101.

Facchini C. (2001): Vincoli e strategie familiari nella scelta del percorso formativo, in Cavalli A. Facchini C. (a cura di), Scelte cruciali. Indagine IARD su giovani e famiglie di fronte alle scente alla fine della scuola secondaria. Bologna: il Mulino, pp. 17-167.

Gambetta D. (1990): Per amore o per forza? Le decisioni scolastiche individuali. Bologna: il Mulino.

Gasperoni G. (1996): Diplomati e istruiti. Rendimento scolastico e istruzione secondaria superiore. Bologna: il Mulino.

Gasperoni G. (2002): I processi formativi fra vecchie disuguaglianze e nuove trasformazioni, in Buzzi C., Cavalli A., De Lillo A. (a cura di): Giovani del nuovo secolo. Quinto rapporto IARD sulla condizione giovanile in Italia. Bologna: il Mulino, pp. 73-96.

Lahaye W., Pourtois J.P., Desmet H. (2007): Transmettre. D'une génération à 
l'autre. Paris : Presses Universitaires de France.

Loeb S., Fuller B., Lynn Kagan S., Carrol B. (2004): Child Care in Poor Communities: Early Learning Effects of Type, Quality, and Stability. Child Development, vol. 75, 1, pp. 47-65.

Lynch R. (2004): Exceptional returns: Economic, fiscal, and social benefits of investment in early childhood development. Washington DC: Economic Policy Institute.

Nimal P., Lahaye W., Pourtois J.P. (2000): Logiques familiales d'insertion sociale. Bruxelles: De Boeck.

Palacio-Quintin E., Terrisse B. (1997): L’environnement familial et le développement de l'enfant d'âge préscolaire. La revue internationale de l'éducation familiale, n. 1, pp. 71-82.

Pan B.A., Rowe M.L., Singer J.D., Snow C.E. (2005): Maternal correlates of growth in toddler vocabulary production in low-income families. Child Development, vol. 76, pp. 763-782.

Pancsofar N., Vernon-Feagans L., Odom-Roe J.R. (2008): Family relationships during infancy and later mother and father vocabulary use with young children. Early Childhood Research Quarterly, n. 23, pp. 493-503.

Pancsofar N., Vernon-Feagans L. (2006): Mother and father language input to young children: Contributions to later language development. Journal of Applied Developmental Psychology, 27, pp. 571-587.

Pourtois J.P. (1979), Comment les mères enseignent à leur enfant (5-6 ans). Paris: Presses Universitaires de France.

Pourtois J.P., Barras C. (2004): Rencontres parentales en milieu défavorisé: le travail sur l'estime de soi. La revue internationale de l'éducation familiale, n. 1, pp. 57-70.

Pourtois J.P., Desmet H. (2004): L'éducation implicite. Socialisation et individualisation. Paris: PUF.

Pourtois J.P., Desmet H., Lahaye W. (2004): Connaissances et pratiques en éducation familiale et parentale. Enfances, Familles, Générations, n. 1.

Pourtois J.P., Desmet H., Lahaye W. (2008): L'implicite des relations, base de l'éducation familiale. Éducation et Sociétés, n. 2, pp. 87-96.

Prêteur Y., De Léonardis M. (2005): Précarités et scolarités. Empan, n. 4, pp. 101-107.

Ravn B. (1996): Current trends in political and pedagogical conditions for family community and school partnershin in Europe, in Winther Jensen T. (a cura di), Challenges to European Education: cultural values, national identities and global responsabilities. Berne: Peter Lang.

Rebello Britto P., Brooks-Gunn J.-M., Griffin Source T.M. (2006): Maternal Reading And Teaching Patterns: Associations With School Readiness In Low-Income African American Families. Reading Research Quarterly, vol. 41, 1, pp. 68-89.

Reynolds A.J., Temple J.A., Robertson D.L. Mann E.A. (2001): Long-term effects of an early childhhod intervention on educational achievement and 
juvenile arrest: a 15 year follow-up of low-income children in public schools. Journal of the American Medical Association, 285, 18, pp. 2339-2346.

Russell Carter D., Chard D.J. Lull Pool J. (2009): A Family Strengths Approach to Early Language and Literacy Development. Early Childhood Educational Journal, vol. 36, 6, pp. 519-526.

Swick K.J. (2009): Promoting School and Life Success Through Early Childhood Family Literacy. Early Childhood Educational Journal, vol. 36, 5, pp. 403-406.

Tamis-LeMonda C.S., Shannon, J.D., Cabrera, N.J., Lamb M.E. (2004): Fathers and mothers at play with their 2 - and 3 -year-olds: Contributions to language and cognitive development. Child Development, vol. 75, pp. 1806-1820.

Varin D. (2007): L'esperienza precoce ed estesa di asili nido: fattori di facilitazione per lo sviluppo e aspetti di rischio. Psicologia clinica dello sviluppo, n. 3, pp. 359-383

Votruba-Drzal E., Levine Coley R., Lindsay Chase-Lansdale P. (2004): Child Care and Low-Income Children's Development: Direct and Moderated Effects. Child Development, vol. 75, 1, pp. 296-312.

Zaouche-Gaudron C. (2005): Les conditions de vie défavorisées influent-elles sur le développement des jeunes enfants. Ramonville Saint-Agne: Eres.

Zaouche-Gaudron C. (2006): Prévention et rapports de l'enfant aux autres et aux milieux de vie, in G. Neyrand et alii (a cura di): Familles et petite enfance. Mutations des savoirs et des pratiques. Ramonville Saint-Agne: Eres, pp. 225-237. 